\title{
Calculation of Residual Magnetic-Field Distributions upon Hysteretic Interference of a Pulsed Magnetic Field
}

\author{
V. V. Pavlyuchenko, E. S. Doroshevich*, and V. L. Pivovarov \\ Belorussian National Technical University, pr. Nezavisimosti 4, Minsk, 220030 Belarus \\ *e-mail: es_doroshevich@mail.ru \\ Received June 16, 2014
}

\begin{abstract}
Equations that are used for the calculation and distributions of the electrical voltage $U(t)$ output from a magnetic-field transducer as it scans a discrete magnetic medium with recorded residual magnetic fields, which were produced in it by acting individual magnetic-field pulses with oppositepolarity surges, are reported. The distributions were obtained via the Delphi programming language. The phenomenon of hysteretic interference $(H I)$ of a magnetic field, which allows one to increase the accuracy of measurements, is used for the inspection of objects made from electrocoductive and magnetic materials.
\end{abstract}

Keywords: magnetic-field strength, tangential component of the magnetic-field strength, specific electrical conductivity, magnetic permeability, magnetic carrier, inductive magnetic head

DOI: $10.1134 / \mathrm{S} 1061830915010076$

\section{List of Designations}

$\sigma$ is the specific electrical conductivity;

$\mu$ is the relative magnetic permeability;

$H$ is the magnetic-field strength;

$H_{\tau}$ is the tangential component of the magnetic field;

$H_{\tau m}$ is the maximum magnitude of $H_{\tau}$;

$H_{n m}$ is the maximum magnitude of the normal magnetic-field component;

$t_{\max }$ is the magnetic-field pulse rise time;

$d$ is the thickness of an object;

MM is a magnetic medium;

MH is a magnetic head;

IMFT is an incremental magnetic-field transducer;

$U$ is the electrical voltage;

$H I$ is the hysteretic interference of a magnetic field.

The aim of the present study is to develop a hysteretic interference method for the inspection of objects that are made from electoconductive and magnetic materials, which increases the accuracy of determination of their specific electrical conductivity $\sigma$, magnetic permeability $\mu$, uniformity of $\sigma$ and $\mu$ distributions, thickness, and parameters of discontinuity flaws in the materials. In this case, the development of the aforementioned method is realized via the application of digital calculations that are performed with the Delphi programming language.

The phenomenon of hysteretic interference of the magnetic field, which consists in the appearance of regular maxima and minima in the distributions of remanent magnetization of a magnetic medium that result from the action of different-polarity magnetic-field pulses applied to it, was considered in [1-3]. The possibilities of the selection of electroconductive objects based on the time constant of the transition process in applying rectangular magnetic-field pulses to an object were discussed in [4], in which a transition-process method was used and the induction flux through a test object was calculated.

The remanent magnetization distribution in a MM depends on its magnetic properties, parameters of pulsed magnetic field, and spatial distribution of $H_{\tau m}$ and $H_{\tau n}$ components of applied field. In this case, both a single alternating-polarity pulse and several single magnetic-field pulses with alternating polarity 
and decreasing amplitude can be applied. As the primary magnetic-field source, we used a linear inductor; as the MM, magnetic-field transducers manufactured from different types of magnetic ribbons were used.

The IMFTs were manufactured from the MM. They consist of parallel magnetic strips that have the same thickness, which are mounted on a flexible nonmagnetic base and are equally spaced from each other. Because of the high anisotropy of magnetic properties of the MM along its plane and perpendicular to the plane, the tangential component of the magnetic-field strength was recorded with the aforementioned IMFTs.

The scanning of the MH with the IMFT was done perpendicular to the magnetic strip axis. The MH output was connected with the digital oscillograph input; the oscillograph was connected to a monitor. In the course of these experiments, the $U$ magnitude induced by a MH was measured. The $H_{\tau m}$ magnitude was determined using the calibration characteristics of the IMFTs.

The IMFTs allow one to determine the absolute magnitude of magnetic-field strength, which is proportional to the amplitude of voltage pulses at the edges of IMFTs. The width of the magnetic strips used in IMFTs is $2 \times 10^{-4}$ and $3 \times 10^{-4} \mathrm{~m}$ and can be equal to several tens of atomic distances. The IMFTs can be manufactured from various magnetic materials that exhibit hysteretic properties, in particular, from magnetooptical films or flux probes. Information that is recorded in them can be read with polarized and unpolarized light, respectively.

When performing the measurements, the individual properties of IMFTs at all their points were taken into account.

The use of IMFTs allows one to double the magnitude of measured voltage by reading the signal from both sides of the magnetic strips and to obtain the distribution of the voltage with an automatically controlled zero signal level. The sensitivity of hysteretic magnetic-field transducers is substantially higher than that of an analogous anhysteretic transducer because of the high slope of the initial portion of the inverse $U(H)$ dependence.

When setting parameters of consecutive pulses and applying them to objects, information about the properties of the objects, namely, voltage $(U)$ distributions at surface points and along a given measuring line $x$, can be obtained.

The measurements were performed at magnetic field strengths of from $1 \times 10^{2}$ to $1 \times 10^{5} \mathrm{~A} / \mathrm{m}$ and a magnetic-field strength rise time of from $1 \times 10^{-6}$ to $5 \times 10^{-4} \mathrm{~s}$ using an experimental setup [3].

The magnetic-field pulse modes produced by the setup are a half-wave pulse, a quarter-wave pulse (exponent), a half-wave pulse with a single inverse magnetic-field $(H)$ surge, a half-wave pulse with several inverse magnetic-field $(H)$ surges, a linearly rising pulse, a trapezoidal pulse, a pulse consisting of three linear portions that differ in slope, and other pulses.

We applied a magnetic-field pulse on the transducer, which is produced by a linear inductor that is located parallel to the transducer plane at a distance of $6 \times 10^{-3} \mathrm{~m}$ from the transducer surface. The tangential component of magnetic-field strength along a line perpendicular to the inductor axis along the $x$ line is calculated by the expression

$$
y=A\left(x^{2}+0.36\right)
$$

where $y=H_{\tau}$. In this case, $x$ and $y$ are measured in $\mathrm{cm}$ and $\mathrm{A} / \mathrm{cm}$, respectively. Figure $1(1)$ shows the dependence at $A=360$.

When reading the information from the MM with the magnetic-field transducer, the dependence of $U$ at the transducer output is obtained, which corresponds to curve 1 in the case of the linear dependence $U=U(H)$ of the calibration characteristic of the magnetic medium. It should be specified that the characteristic is the remanent magnetization of the magnetic medium. In fact, the dependence $U=U(H)$ is nonlinear. Figure 2 shows such a dependence for the used MM.

The dependence $U=U(H)$ was obtained during direct magnetization of an MM. In order to calculate the remanent magnetization of areas of an MM that were subjected to the action of the magnetic-field pulse produced by a linear inductor, the dependence $U=U(H)$ is divided into six linear portions. For the first, initial, portion of the curve, the voltage output from the magnetic-field transducer is zero:

$u 1=0$; in the magnetic field strength range $0<y<30$, where $y(H)$ is measured in $\mathrm{A} / \mathrm{cm}$.

For the second portion, the voltage magnitude output from the magnetic-field transducer is determined by expression

$u 2=0.222(y-30)$; in the field strength range $30<0<120$.

For the third portion:

$u 3=20+0.25(y-120)$; in the range $120<y<200$. 


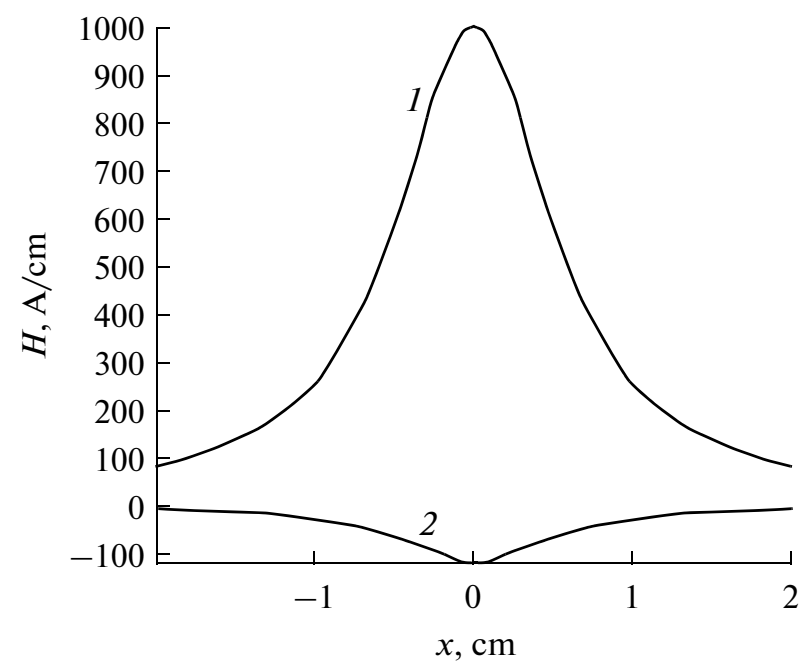

Fig. 1. The dependences of the tangential component of magnetic field strength on the $x$ distance to the projection of linear inductor axis.

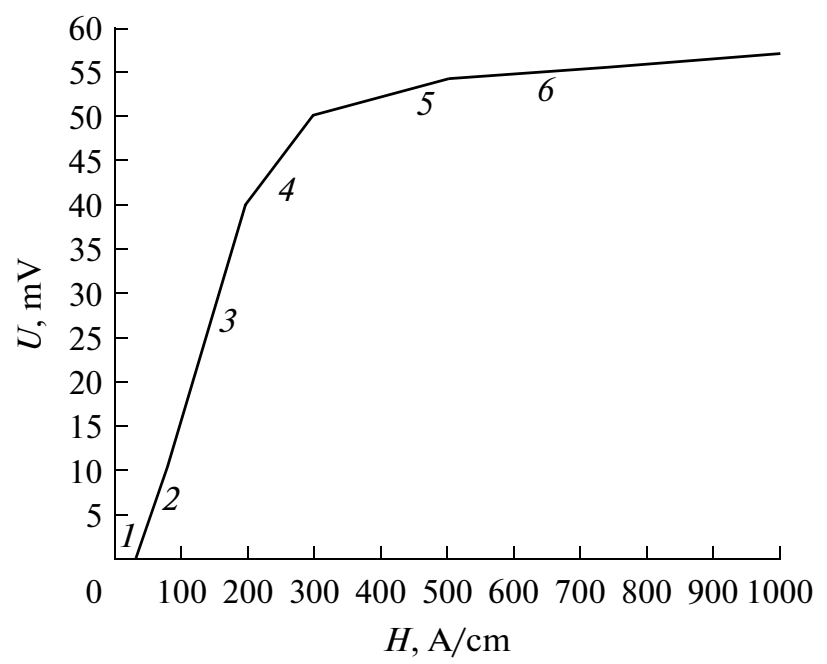

Fig. 2. The dependence (direct) of the voltage magnitude, $U$, output from the magnetic-field transducer on the magnetic field, $H$, applied to an MM.

For the fourth portion:

$u 4=40+0.1(y-200)$; in the range $200<y<300$.

For the fifth portion:

$u 5=50+0.02(y-300)$; in the range $300<y<500$.

For the sixth portion:

$u 6=54+0.006(y-500)$; in the range $500<0 y<1000$.

We apply the second magnetic-field pulse produced by linear inductor on the magnetic-field transducer, which is retudrning and has the strength magnitude equal to

$$
y 1=B /\left(x^{2}+0.36\right) \text {. }
$$

In this case, $x$ and $y 1$ are measured in $\mathrm{cm}$ and $\mathrm{A} / \mathrm{cm}$, respectively. Figure 1 (2) shows the dependence at $B=-43$.

To calculate the $U=U(x)$ distribution, which results from the second magnetic-field pulse applied to the magnetic-field transducer, we use the experimental dependence $U=U(H)$ obtained after magnetization reversal of an MM from states that are characterized by different remanent magnetizations that were determined in accordance with the parameters of the direct-magnetization dependence. Figure 3 shows one such inverse dependence $U=U(H)$ consisting of two linear portions. The points of the transition of 


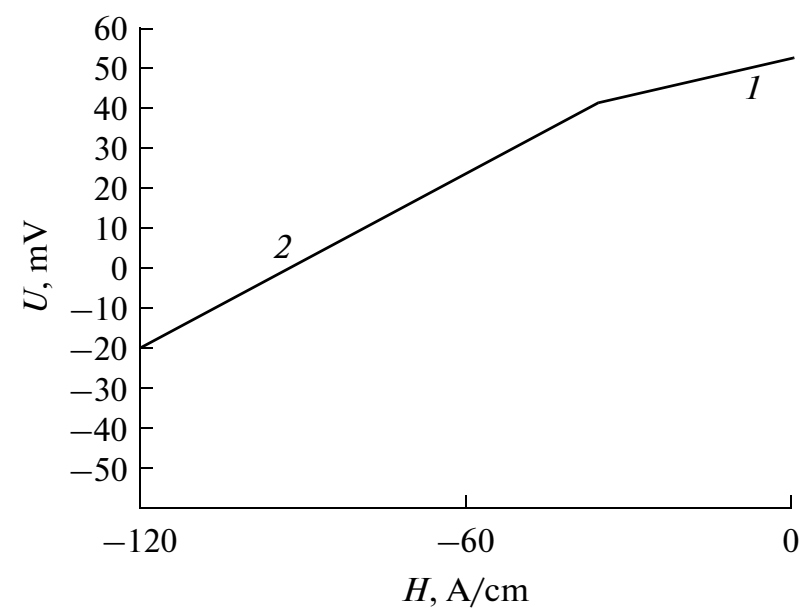

Fig. 3. The dependence (inverse) of the voltage magnitude, $U$, output from magnetic-field transducer on the field magnitude, $H$, applied to an MM.

one linear dependence into the other were obtained at $y 1=-30$; the $U$ magnitude at these points is 0.8125 of the $U$ magnitude that is reached upon direct magnetization.

The dependences $U=U(x)$, which correspond to portions $u 2-u 6$ in the initial dependence $U=U(H)$ at $y 1<-30$ was calculated by the following functions:

$u 6=54+0.006(y-500)$

$u 19=0.8125 u 6$

$u 14=u 19+(y 1+30)(u 19+20) / 90 ;$ in the range $0<x<0.6 ;$

$u 6=54+0.006(y-500)$

$u 19=0.8125 u 6$

$u 14=u 19+(y 1+30)(u 19+20) / 90 ;$ in the range $-0.6<x<0 ;$

$u 5=50+0.02(y-300)$

$u 18=0.8125 u 5$

$u 13=u 18+(y 1+30)(u 18+20) / 90 ;$ in the range $0.6<x<0.917$;

$u 5=50+0.02(y-300)$;

$u 18=0.8125 u 5$;

$u 13=u 18+(y 1+30)(u 18+20) / 90 ;$ in the range $-0.917<x<-0.6$;

$u 4=40+0.1(y-200)$

$u 17=0.8125 u 4$

$u 12=u 17+(y 1+30)(u 17+20) / 90 ;$ in the range $0.917<x<1.2$;

$u 4=40+0.1(y-200)$

$u 17=0.8125 u 4$;

$u 12=u 17+(y 1+30)(u 17+20) / 90 ;$ in the range $-1.2<x<-0.917 ;$

$u 3=20+0.25(y-120)$;

$u 16=0.8125 u 3$;

$u 11=u 16+(y 1+30)(u 16+20) / 90 ;$ in the range $-25<x<0 ;$

$u 3=20+0.25(y-120)$;

$u 16=0.8125 u 3$;

$u 11=u 16+(y 1+30)(u 16+20) / 90 ;$ in the range $1.2<x<1.625 ;$

$u 3=20+0.25(y-120)$

$u 16=0.8125 u 3$;

$u 11=u 16+(y 1+30)(u 16+20 / 90) ;$ in the range $-1.625<x<-1.2$;

$u 2=0.222(y-30)$

$u 15=0.8125 u 2$;

$u 10=u 15+(y 1+30)(u 15+20) / 90 ;$ in the range $-3.412<x<-1.625$. 


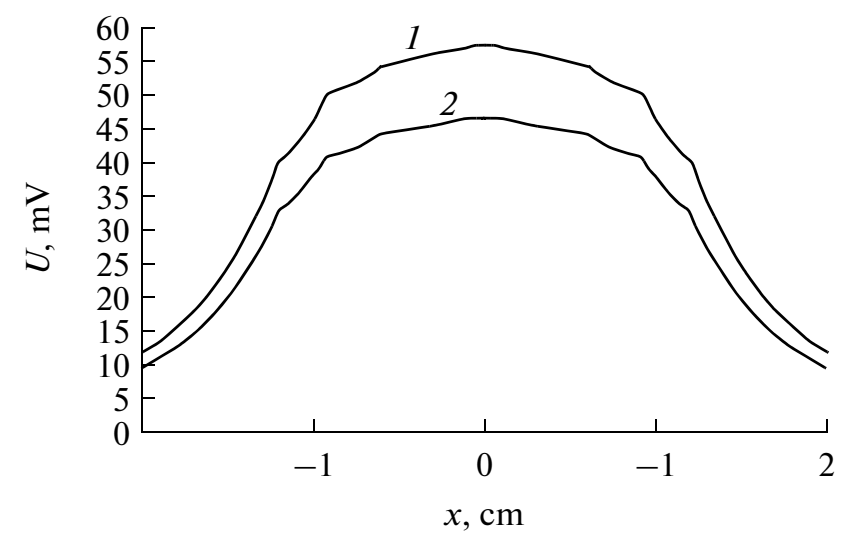

Fig. 4. The dependence of the voltage magnitude, $U$, output from the magnetic-field transducer on the distance, $x$, to the projection of linear inductor axis.

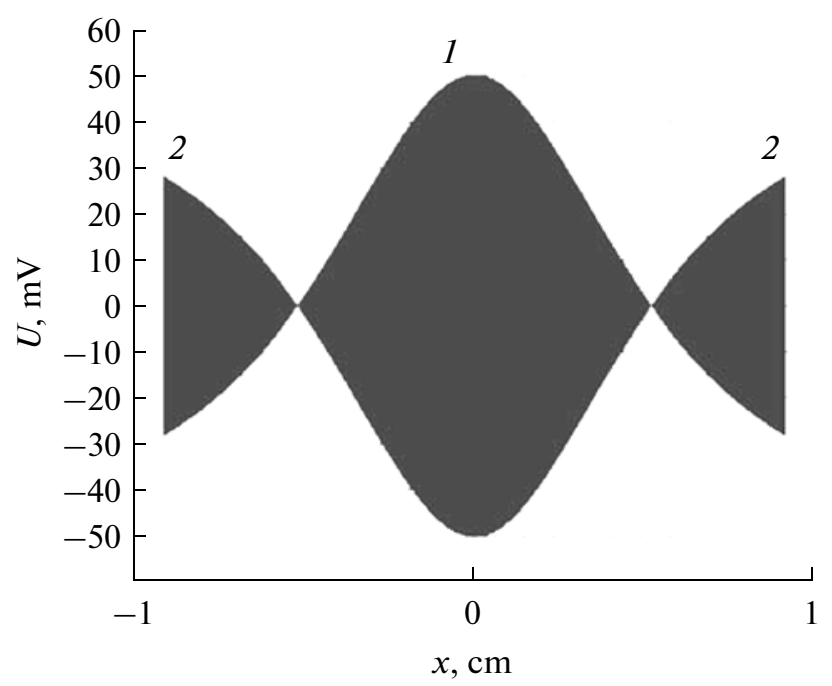

Fig. 5. The dependence of the voltage magnitude, $U$, output the magnetic field transducer on the distance, $x$, to the projection of linear inductor axis; $U_{m}=100 \mathrm{mV}$.

Figure 4 (1) shows the voltage distributions that correspond to the functions $u 10-u 14$.

These distributions result from the sequential action of the $y$ and $y_{1}$ field pulses on the magnetic medium. Figure 4 also shows the dependence 2, which includes functions $u 15-u 19$. In order to obtain theoretical distributions $U=U(x)$ analogous to the experimental dependences $U=U(x)$, flipped dependences $u 10-u 14$ should be plotted. Such calculated bipolar distributions are given in Fig. 5 (direct function $(a=360, B=-58)$ and flipped function $(A=-360, B=58), 6$ (direct function $(A=360, B=-43))$ and flipped function $(A=-360, B=43))$, and 7 (direct function $(A=360, B=-33.5)$ and flipped function $(A=-360, B=33.5))$.

The aforementioned distributions allow us to double the signal amplitude and to automatically set the zero-signal level; due to these circumstances, the accuracy of measurement of the signal maxima increases.

The maxima and minima are numbered in Figs. 4-7. In finding experimental data and performing theoretical program calculations of magnetic-field and voltage distributions, operations such as the separation of portions of dependences, zero signal level shifting, and signal amplification for these portions, which is followed by obtaining new interference patterns, should be performed. Figures 8-11 show the sequence of such operations that are performed in the course of theoretical calculations of distributions $U=U(x)$ corresponding to residual magnetic-field distribution of the MM, in which local maxima and minima of signals are numbered. The sequence of operations includes the separation of the portion of dependence under the inductor axis (Fig. 8), 6-fold amplification of the signal and a shift of its zero level 


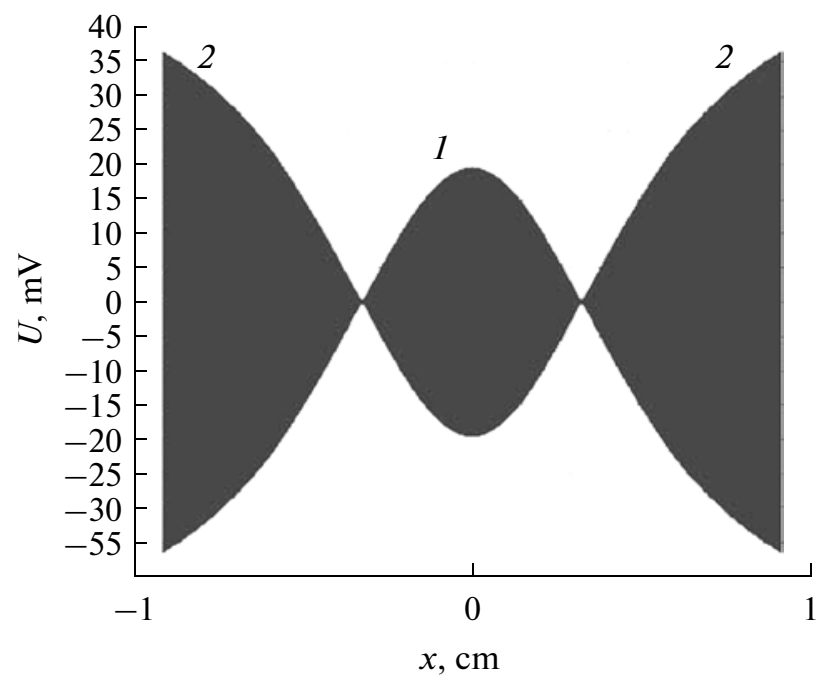

Fig. 6. The dependence of the voltage magnitude, $U$, output the magnetic field transducer on the distance, $x$, to the projection of linear inductor axis; $U_{m}=40 \mathrm{mV}$.

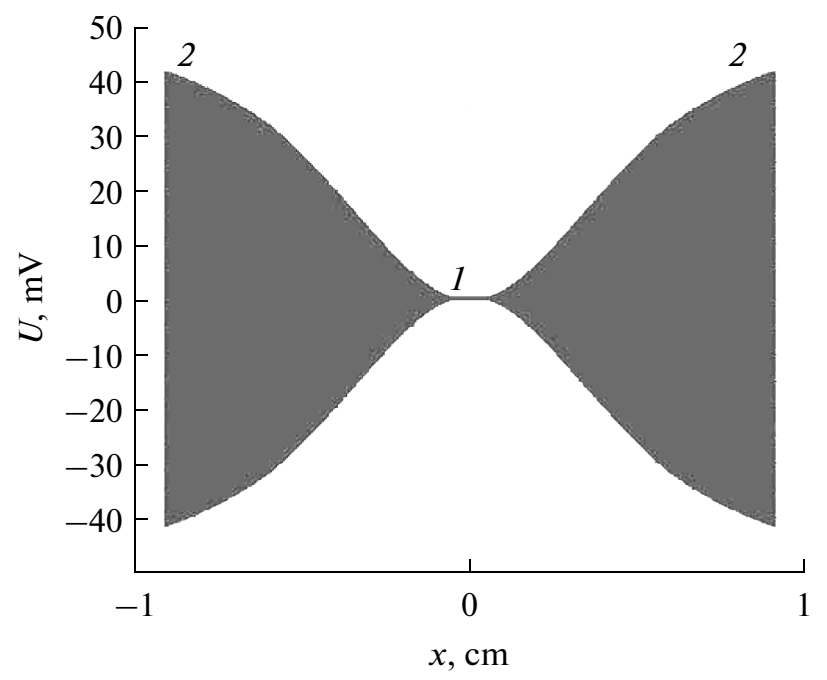

Fig. 7. The dependence of the voltage magnitude, $U$, output the magnetic field transducer on the distance, $x$, to the projection of linear inductor axis; $U_{m}=0 \mathrm{mV}$.

by $12 \mathrm{mV}$ (Fig. 9), plotting direct and flipped functions for a new zero signal level (Fig. 10), and formation of an optical image (new interference pattern) using direct and flipped functions (Fig. 11).

Using the developed procedure and methods, we can calculate the distributions $U_{n}=U_{n}(x)$, which will result from the action of magnetic-field pulses on the MM that is located on the surface of an object made from an electroconductive material and can determine the properties of the object via the comparison of the obtained distributions with the reference ones. When returning to the data of [3], we can conclude that the thickness of metallic plate can be determined to a high accuracy based on the central maximum magnitude. In particular, the increase in the voltage from $U_{m}=40$ to $U_{m}=100 \mathrm{mV}$ corresponds to the logging range of $60 \mathrm{mV}$. The limit of the relative error is determined by the expression $\delta= \pm \frac{\Delta \times 100}{x} \%$, where $\Delta$ is the limit of the absolute error. When the limit is equal to $1,0.1$, and $0.01 \mathrm{mV}$, the relative errors are $1.7,0.17,0.017 \%$, respectively. The accuracy of the measurements increases by many times via the use of the initial hysteretic portion, with low $H$ magnitudes, of the inverse calibration dependence for an MM (Fig. 3, portion 1), for which $U=0$ in the case of anhysteretic calibration dependence (initial dependence, Fig. 2, portion 1 ). 


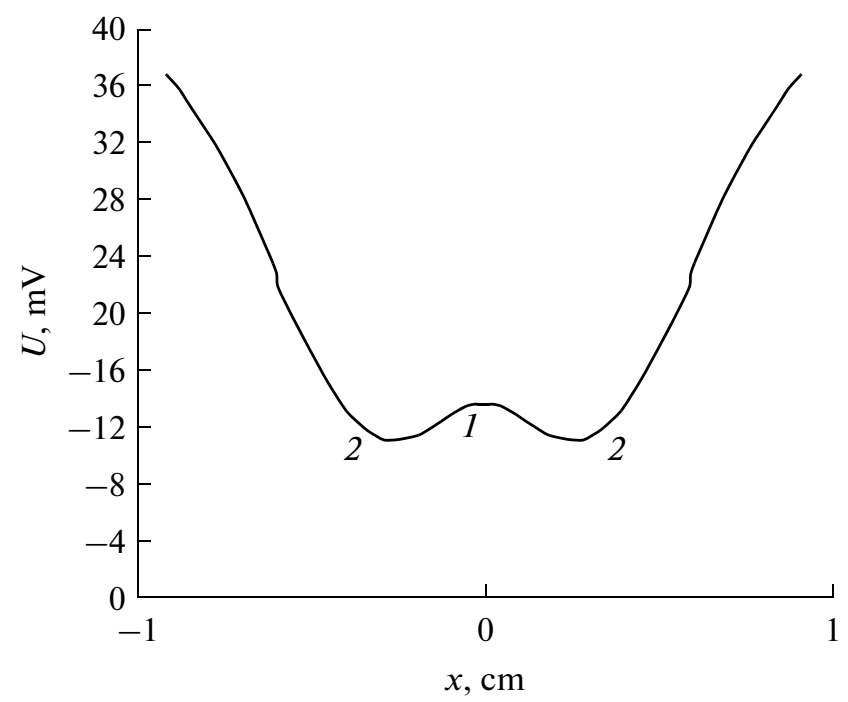

Fig. 8. The dependence of the voltage magnitude, $U$, output the magnetic field transducer on the distance, $x$, to the projection of linear inductor axis.

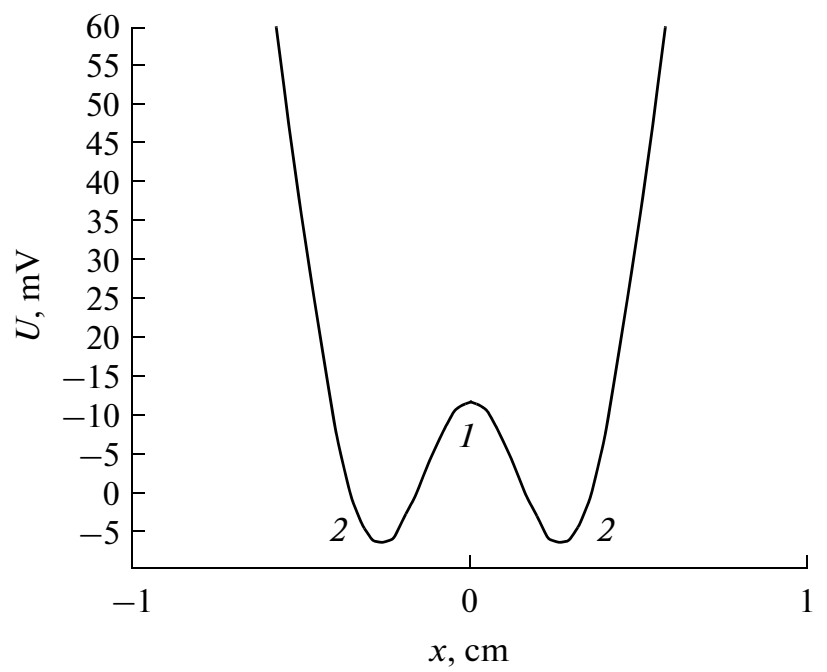

Fig. 9. The dependence of the voltage magnitude, $U$, output the magnetic field transducer on the distance, $x$, to the projection of linear inductor axis.

In the case of theoretical calculations, the increase in the accuracy of determination of parameters of magnetic fields by using $H I$ is inexhaustible; in practice, the increase is multifold. The $H I$ method assumes the application of any nonuniformity in the magnetic-field distribution and, in the case of the absence of nonuniformity, the artificial creation of such nonuniformities. The method can be applied to any hysteretic phenomena, such as magnetic, electrical, mechanical, and optical phenomena. This allows one to instantly record the magnetic-field distribution over considerable surface areas of different shapes; the use of a magnetic-field transducer operating in real time, for example, of magnetooptical film or flux-probes, allows one to instantly read the magnetic-field information without intermediate recording.

Thus, the sequence of operations of performed programmed calculations of $H I$ is as follows:

(1) The choice of an experimental (theoretical) dependence $U=U(H)$ for a concrete MM.

(2) Representation of the dependence in the form of several functions in terms of a given approximation $U_{i 1}=U_{i 1}(H)$.

(3) Determination of the parameters of the first acting direct pulse, such as the current magnitude of a linear inductor, current pulse rise and decay times, pulse shape, and the spacing between the linear inductor axis and MM. 


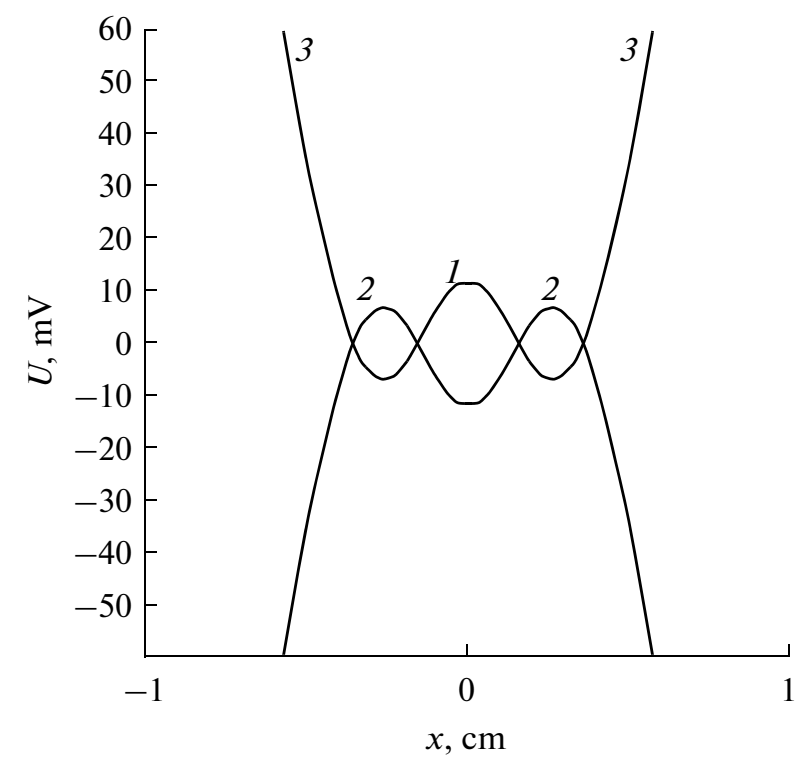

Fig. 10. The dependence of the voltage magnitude, $U$, output the magnetic field transducer on the distance, $x$, to the projection of linear inductor axis.

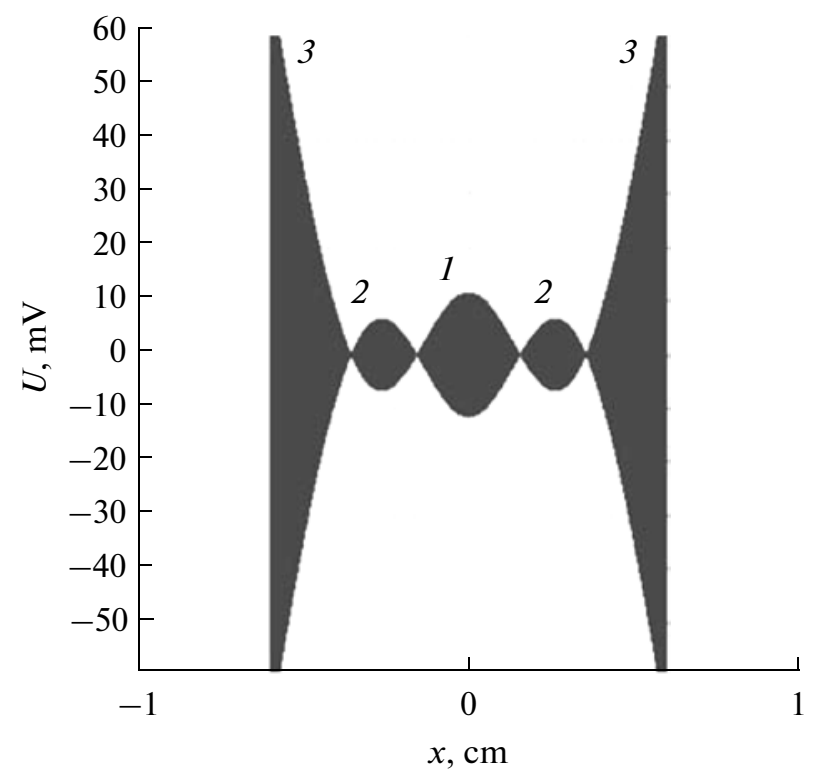

Fig. 11. The dependence of the voltage magnitude, $U$, output the magnetic field transducer on the distance, $x$, to the projection of linear inductor axis.

(4) Calculation of the effect of the found magnetic-field pulse on the MM and determination of the dependence $U=U(t)$ when the magnetic-field transducer reads a signal along the given measuring line of the MM.

(5) Transformation of the time dependence $U=U(t)$ into the spatial dependence $U_{1}=U_{1}(x)$ along the reading coordinate $x$ in accordance with the transformation ratio.

(6) Calculation and plotting of the dependence $U_{2}=U_{2}(x)$ by application of the calibration functions $U_{i}=U_{i}(H)$ for the dependence $U_{1}=U_{1}(x)$.

(7) Determination of the experimental (theoretical) inverse calibration dependences $U_{j}=U_{j}(H)$ for the used MM. 
(8) Representation of the dependences in the form of several functions within the given approximation $U_{i 2}=U_{i 2}(H)$.

(9) Determination of the parameters of the second applied returning pulse, such as the current of a linear inductor, pulse rise and decay time, pulse shape, and spacing between the linear inductor axis and MM.

(10) Calculation of the effect of the second magnetic-field pulse on the MM and plotting the dependence $U_{2}=U_{2}(x)$, which results from the action of two magnetic-field pulses on the MM, when reading the signal with the magnetic-field transducer along a given measuring line of an MM and using the dependences $U_{j}=U_{j}(H)$ and $U_{i 2}=U_{i 2}(H)$.

(11) Calculation of the effect of the subsequent found magnetic-field pulses and plotting analogous resulting dependences $U_{n}=U_{n}(x)$.

(12) Shifting the zero signal level when an increase in the accuracy of measurements is necessary.

(13) Collective plotting direct and flipped distributions with amplifying signals.

(14) Formation of optical images with maxima and minima of signals.

(15) Determination of the experimental distributions of $H I$ and optical images in accordance with the calculated data for a concrete reference object.

(16) Choice of a test object, repeated execution of items 1-14 in the presence of the test object and determination of its properties via the comparison of the obtained distributions $U_{n}=U_{n}(x)$ and optical images with analogous distributions and images for the reference object.

Using the developed software, these calculations are performed instantly as well.

\section{CONCLUSIONS}

(1) The results of calculations of the distributions of the voltage $U(t)$ output from a magnetic-field transducer during hysteretic pulse magnetic-field interference $(H I)$ are given. The distributions $U(t)$ correspond to field distributions $H(x)$ along coordinate $x$, which are recorded on a magnetic medium. Individual magnetic-field alternating-polarity pulses were applied to the MM. The calculations were performed using the Delphi programming language.

(2) It was shown that the phenomenon of hysteretic interference of pulsed magnetic field, developed methods of measurement, control devices, and magnetic-field transducers can be used for the measurement of momentary distributions of the magnetic-field strength at any point in space and on a surface of any shape and, in the case of the application of magnetic-field transducers, for instant reading, without intermediate recording, of magnetic-field information.

(3) The performed theoretical calculations of the $H I$ phenomenon allow one to increase the accuracy of the determination of magnetic-field distributions, measurements of the thickness of electroconductive objects and determination of their electrical conductivity $\sigma$ and magnetic permeability $\mu$, uniformity of $\sigma$ and $\mu$ distributions, and the parameters of discontinuity flaws in the objects as well.

\section{REFERENCES}

1. Pavlyuchenko, V.V. and Doroshevich, E.S., Odnim impul'som (With Single Pulse), Saarbrucken: LAP LAMBERT Academic Publishing, 2013.

2. Pavlyuchenko, V.V. and Doroshevich, E.S., Nondestructive Control of Objects Made of Electroconductive Materials in Pulsed Magnetic Fields, Rus. J. Nondestr. Test., 2010, vol. 46, no. 11, pp. 810-818.

3. Pavlyuchenko, V.V. and Doroshevich, E.S., Using Magnetic Hysteresis for Testing Electroconductive Objects in Pulsed Magnetic Fields, Rus. J. Nondestr. Test., 2013, vol. 49, no. 6, pp. 334-346.

4. Reutov, Yu.Ya. and Litvinenko, A.A., On the possibility of selection of electroconductive objects based on the time constant of transition process upon jump-like variation of magnetizing field, Defektoskopiya, 1991, no. 10, pp. 80-87.

5. Flenov, M., Bibliya Delphi (Delphi Bible), St. Petersburg: BKhV-Peterburg, 2011.

Translated by N. Kolchugina 